\title{
Preserving Access and Increasing Discovery of State-Level Geoscience Grey Literature
}

Paper 186-4 Booth 75

Leila M. Gonzales*, Christopher Keane, Sharon Tahirkheli. American Geosciences Institute, Alexandria, VA. * corresponding author: Img@americangeosciences.org

What is grey literature?

Grey literature is factual documents "produced on all levels of government, academics, business and industry in electronic and print formats not controlled by commercial publishing, i.e. where publishing is not the primary activity of the producing body"., 2,

Examples include: technical reports, fact sheets, case studies, monographs, conference proceedings, working papers, guides, position papers, etc.

State geological surveys are the primary producer of state-level geoscience grey literature.

Why is grey literature important?

Grey literature fills the gaps in scientific knowledge by corroborat-

ing conclusions found in peer-reviewed scholarly journals, and in

many cases provides unique, location-specific or detailed applied science information.

Grey literature is aimed at informing policy making, sharing case studies of successful outcomes, translating scientific knowledge for non-scientific audiences, and increasing access to research and information in a timely fashion. ${ }^{3,4}$

Issues with access, discovery, and preservation Lack of long-term access

Lack of adequate bibliographic information

Lack of full-text searching

Overwriting or removal of online information

The loss of access to online geoscience literature is approximately $8 \%$ per year (source: AGI's GeoRef Information System)
Availability of Publications

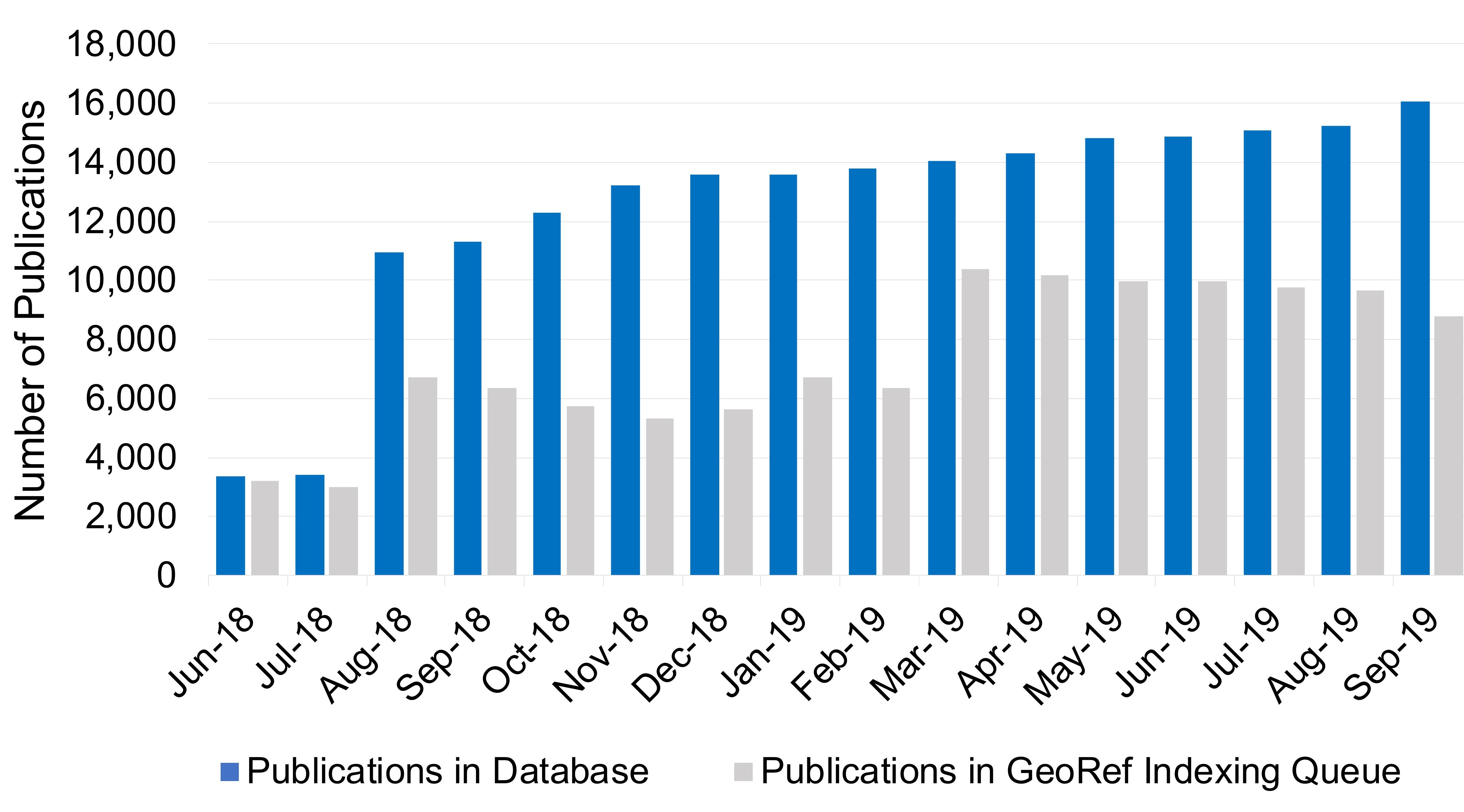

A growing community resource

Coverage of state geological survey publications in the Geological Surveys Database is continually expanding as new publications are indexed by GeoRef staff and made available for searching in the database. New records are added and metadata is updated on a weekly basis. Links to publications and state geological survey websites are checked and updated on a monthly basis.

Metadata for new or updated records are first matched to corresponding bibliographic metadata from GeoRef and are supplemented with additional metadata from the state geological surveys if available. PDF copies of publications are either downloaded from the corresponding state geological survey publication catalog or are provided directly to AGI and then processed for full-text indexing. Next the updated metadata and the full-text of the publications are loaded to the Geological Surveys Database and are made available for searching.
Coverage of State Geological Survey Publications
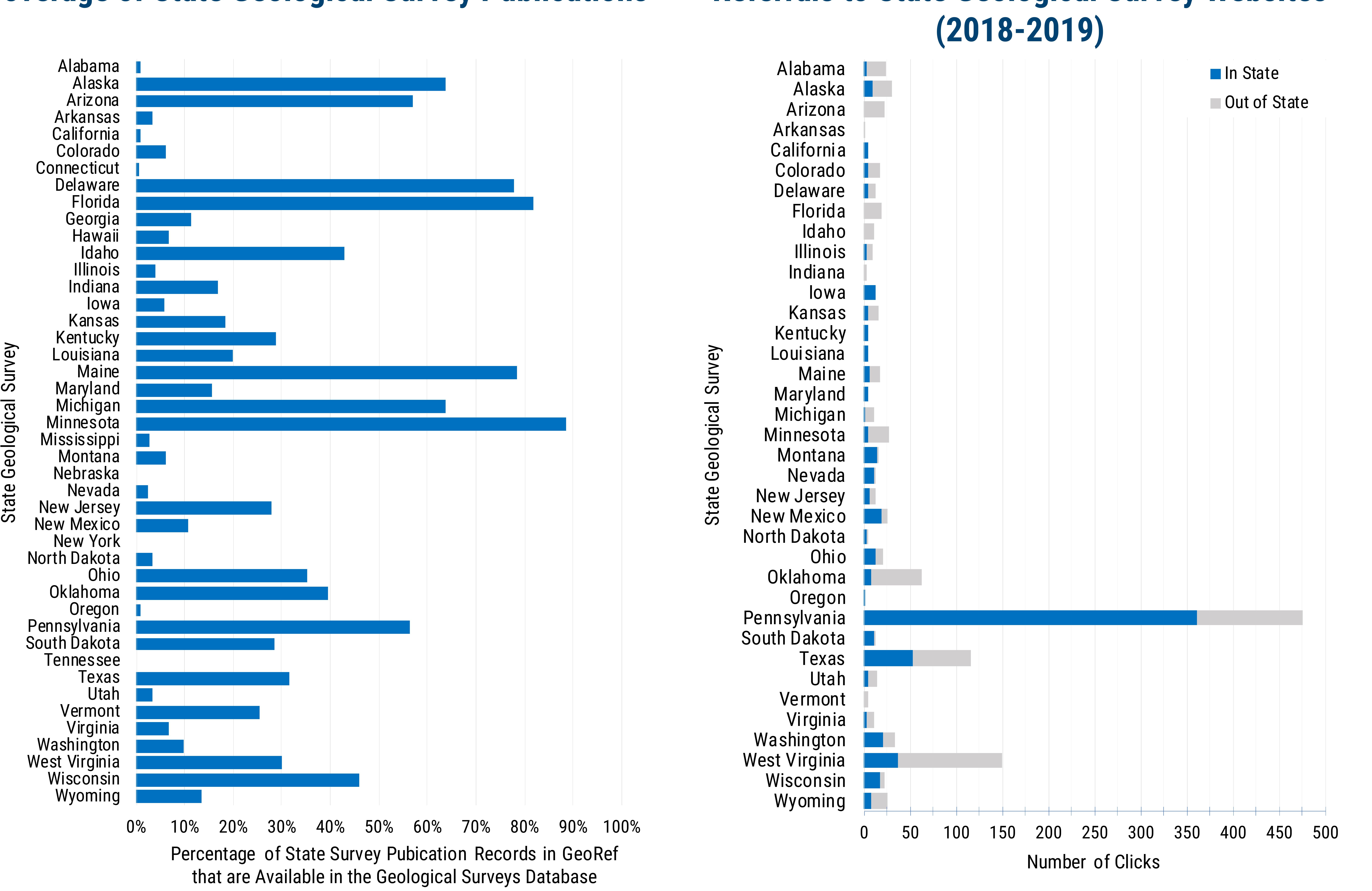

Strategies \& Strengths

- Federated searching across all state geological surveys

- Links to state geological survey websites for publication access

- Integration with GeoRef and dedicated support for long term maintenance of database

- Robust metadata, including geographic coordinates

Multi-faceted searching (full-text, faceted, fuzzy, geographic)

- Ability to serve as a back-up repository if needed

- Integration with other high-value web content on AGI website to increase exposure

https://statesurveys.americangeosciences.org

This database is supported by AGI's GeoRef Information Services and the American Association of State Geologists 